\title{
A deformable mirror based on electrocapillary actuation of a liquid surface
}

\author{
Edgar M. Vuelban, Nandini Bhattacharya, and Joseph Braat \\ Optics Research Group, Department of Imaging Science and Technology \\ Delft University of Technology Delft, The Netherlands
}

\begin{abstract}
We propose and demonstrate a novel liquid based deformable mirror (LDM). The proposed LDM consists of an array of vertically oriented open capillary channels immersed in a pool of two immiscible liquids. A free-floating thin reflective membrane serves as the reflecting surface. By means of jet action, membrane deformation is induced. The control of jet flow through each channel is achieved by electrostatic means. This individual control enables the generation of complex surface profiles useful for adaptive optics applications. The advantages of this device include high stroke dynamic range, low power dissipation, high number of actuators, fast response time, and reduced fabrication cost. The device, however, can only be operated in a vertical orientation and is suitable for dynamic wavefront correction. A proof of principle of the device using an array of linearly addressed capillary channels is presented. Preliminary measurements showed that the response time is several milliseconds with a stroke of more than 10 microns. The design and fabrication of a prototype with around 100 actuators is in progress.
\end{abstract}

Keywords: deformable mirror, high order correction, liquid jet, electrocapillary

\section{INTRODUCTION}

Though deformable mirrors (DM) exist for a few decades now, none of these working DMs are based on liquid (except for the liquid crystal spatial light modulator). Conventional deformable mirrors are based on a thin plate with the actuators attached at the back of this plate. The state-of-the-art solid mechanical deformable mirrors are based on clamped membranes actuated by various means (i.e. electrostatic, piezoelectric, electromagnetic).

It is worth mentioning that the first proposal for correcting atmospheric distortion was based on a liquid system - the Eidophor. ${ }^{1}$ The Eidophor projection system ${ }^{2}$ consists, along with other important optical components, of a thin oil film covering a reflecting mirror. An electron gun bombards electric charges onto the surface of the oil and by means of an electrostatic force a controlled film deformation is achieved. However, no concrete implementation has been reported with this method. At present, only two liquid-based deformable mirrors have been demonstrated. These liquid-based DMs are electromagnetically actuated..$^{3,4}$

There is a growing demand for deformable mirrors with better specifications compared to the conventional DMs - namely large number of actuators, very narrow actuator pitch, low power requirement, and large stroke dynamic range. The planned $100 \mathrm{~m}$ class telescope, for example, needs an estimated number of actuators in the range of $100 k-500 k$ with an interactuator pitch of $<200 \mu \mathrm{m}$ to $1 \mathrm{~mm} .^{5}$

The proposed deformable mirror based on liquid surface actuation offers several advantages. The fabrication of a low cost deformable mirror with a large number of actuators can be easily realized. The fabrication of such a device is compatible with the standard micro-electromechanical systems (MEMS) technology. The scaling of the device to larger dimensions, without posing serious manufacturing problems, is possible by employing a modular design approach. An initial flat surface is easily achieved. The device can generate a high stroke dynamic range. The response time is fast. The device is based on electrostatic actuation and therefore electrical power consumption is minimal. However, like any other device, the proposed LDM has some major drawbacks.

(Send correspondence to E.M.V)

E.M.V.: E-mail: e.vuelban@tnw.tudelft.nl

Advanced Wavefront Control: Methods, Devices, and Applications III, edited by Mark T. Gruneisen,

John D. Gonglewski, Michael K. Giles, Proceedings of SPIE Vol. 5894 (SPIE, Bellingham, WA, 2005)

0277-786X/05/\$15 - doi: 10.1117/12.617248

Proc. of SPIE 58940I-1 


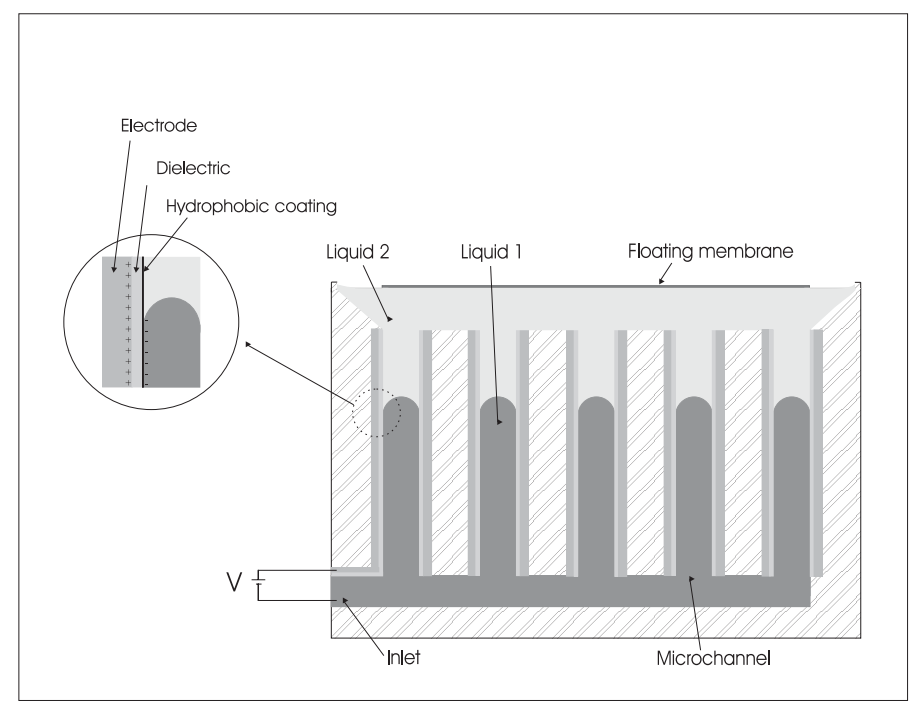

Figure 1. Design concept of an electrostatically actuated liquid-based deformable mirror.

The device can only be operated in a vertical orientation. It is not suitable for static correction and for low-order spatial aberration.

In this paper, we present the principle of the proposed device. A novel way of realizing liquid actuation, based on the electrocapillary effect, is discussed. A first estimation of the response time is presented. The issue of surface waves is discussed in chapter 3 . The proof of principle is presented, as well as the result of preliminary measurements.

\section{DEVICE PRINCIPLE}

The proposed device is shown in Fig. 1. It basically consists of microchannels where capillary surface movement can be realized. The inner wall of each channel is coated with a conducting material, which serves as an electrode, and a thin layer of a dielectric. Liquid 1 is a conducting liquid (e.g., electrolyte) while liquid 2 is a dielectric. The two liquids are immiscible. A free floating reflective membrane is used since there exist only a limited number of highly reflecting liquids. Even then, these liquids are not suitable for a liquid deformable mirror. As liquid 1 flows upward or downward, some volume of liquid 2 is displaced upward or downward, thereby creating a surface bump or a depression. This upward or downward movement of liquid 1 inside the capillary is electrostatically controlled.

These microchannels can be made by etching the substrate through and depositing the electrode and insulating materials on the inner wall. Another possible way of fabricating the device is by processing individual capillaries and attaching them afterward.

\subsection{Electrostatic actuation}

The electrostatic actuation of liquid flow is achieved through the electrocapillary effect. In a classical electrocapillary set-up, ${ }^{6}$ a potential difference between two electrically conducting and immiscible fluids (e.g., mercuryelectrolyte system) results in a modification of the fluid-fluid interfacial tension. This modification of interfacial tension results in a pressure gradient, inside the capillary, that induces a flow of fluid in the channel. However, this phenomenon is not suitable for the proposed device. Electrical current flows and cross-talk between neighboring channels can occur when using two highly conducting liquids such as mercury and an electrolyte. Aside from this, a mercury-electrolyte system has a high density mismatch so that gravity can influence the movement of the fluids inside the capillary. 
For the above mentioned device, the electrocapillary effect is achieved by modifying the interfacial tension of liquid 1 and the dielectric layer. In this way, the flow of electrical current through the channel is prevented. This is basically a capacitor configuration where the conducting liquid serves as a deformable/movable electrode. Leakage current occurs only in the dielectric material. With a suitable dielectric, however, this leakage current is negligible. This technique of liquid actuation inside a capillary was first demonstrated by Welters and Fokkink in $1998 .^{7}$

The amount of electrocapillary pressure that can be generated for a single microchannel is dependent on the diameter of the capillary, the permittivity and thickness of the insulating material, and on the applied voltage. This electrocapillary pressure is expressed as, ${ }^{8}$

$$
\Delta P=\frac{2}{d} \frac{\epsilon_{o} \epsilon_{r}}{\tau} V^{2}
$$

where $\epsilon_{o}$ is the permittivity in vacuum, $\epsilon_{r}$ is the dielectric constant of the insulator, $d$ is the diameter of the capillary, $\tau$ is the thickness of the insulator and $V$ is the applied voltage.

This type of liquid actuation is reversible and the time-scale for the up and down movement of the liquid is of the same magnitude as reported by Welters and Fokkink. ${ }^{7}$

\subsection{Electrocapillary rise}

Since the two liquids are incompressible, the electrocapillary rise time provides a good estimate of how fast the rise time of the surface bump will be when liquid actuation starts. Unlike the conventional capillary rise problem, where the liquid rises when the capillary is in contact with the liquid bath, the liquid column is initially at a certain height inside the capillary (It is only through the application of the electrocapillary force that liquid starts to rise. This situation happens because the inner wall of the capillary is hydrophobic, thereby preventing the liquid to rise by conventional capillary means). The rate of electrocapillary rise can be determined by,

$$
\rho r^{2}\left(y+\frac{r}{2}\right)\left(\frac{d^{2} y}{d t^{2}}\right)+8 \eta y\left(\frac{d y}{d t}\right)+r^{2} \rho g y-\frac{r \epsilon_{o} \epsilon_{r}}{\tau} V^{2}=0
$$

where $y=y(t)$ is the vertical displacement, $\eta$ is the dynamic viscosity of the liquid, $r$ is the radius of the capillary, $\rho$ is the liquid density, and $g$ is gravity. Fig. 2 shows the simulated electrocapillary rise based on Eq. 2. Aside from electrocapillary force, the main forces that govern the dynamics of the rise include inertial, viscous and hydrostatic forces. The influence of the contact line friction associated with molecular kinetics and energy dissipation ${ }^{9}$ was not included in the formulation of Eq 2. In the simulation, a water-air system was considered. A capillary radius of $100 \mu \mathrm{m}$ is used and the dynamic viscosity of water is adjusted to $\eta=7.0 \mathrm{mPa} s$.

\section{SURFACE WAVE}

One of the immediate questions that arise regarding the use of liquid as a deformable medium is the presence of surface waves that would render a deformable mirror unpractical. This fundamental question is addressed in the proposed device.

The dispersion relation for the capillary gravity waves is given by, ${ }^{10}$

$$
f=\frac{\sqrt{\left(g k+\frac{\sigma k^{3}}{\rho}\right) \tanh k h}}{2 \pi}
$$

where $f$ is the frequency, $k$ is the wave number $\left(k=\frac{2 \pi}{\lambda}\right), \sigma$ is the surface tension, and $\rho$ is the density of the top liquid. The first term denotes the gravity wave while the second term is the capillary component. For $k h>>1$, Eq. 3 becomes

$$
f=\frac{\sqrt{\left(g k+\frac{\sigma k^{3}}{\rho}\right)}}{2 \pi}
$$




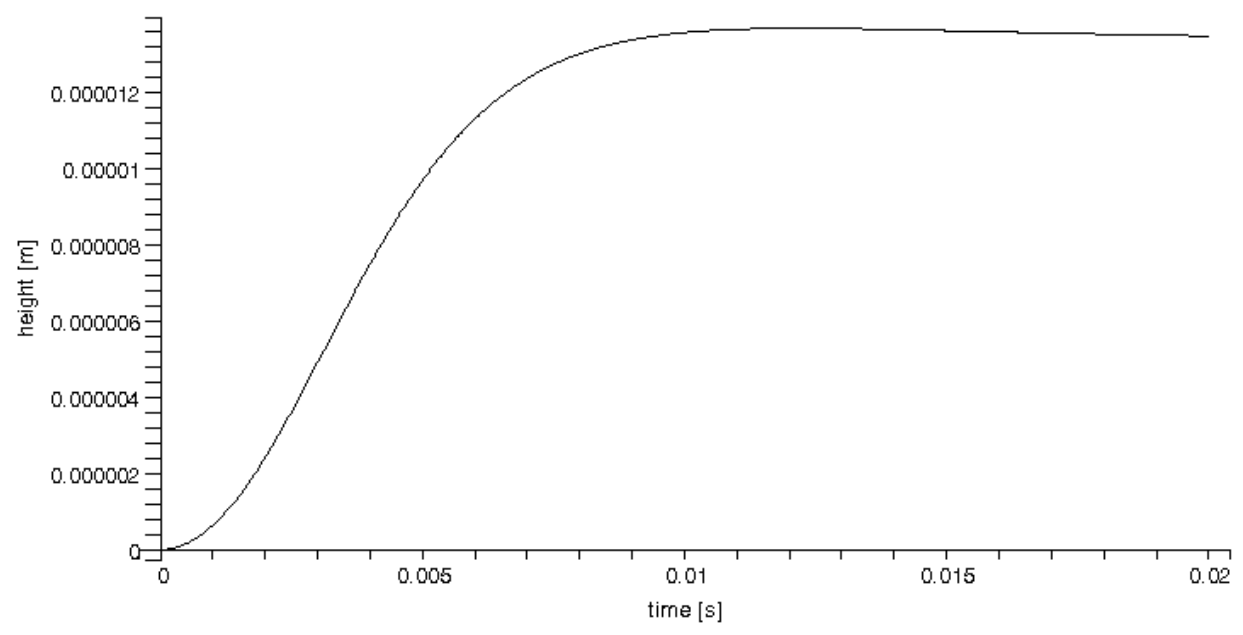

Figure 2. Simulation of the electrocapillary rise, taking into account inertial, viscous, hydrostatic and electrocapillary forces.

For $k h<<1$, Eq. 3 becomes

$$
f=\frac{\sqrt{\left(g h k^{2}+\frac{\sigma h k^{4}}{\rho}\right)}}{2 \pi}
$$

The proposed device has a capillary dimension of a few hundred microns and an actuator pitch of the same order. It is safe to assume then that the wavelength of surface waves is less than a millimeter. With this wavelength, the capillary wave phenomenon dominates over the gravity wave effect and Eq. 4 reduces to

$$
f=\frac{\sqrt{\left(\frac{\sigma k^{3}}{\rho}\right)}}{2 \pi}
$$

The damping coefficient for surface waves is given by,

$$
K=\frac{2 \eta k^{2}}{\rho}
$$

A damped wave is attained when $K=2 \pi f$. At this condition, we can determine the required viscosity of liquid 2 to achieved a damped wave. Overdamping occurs when $K>2 \pi f$. The required viscosity is expressed as

$$
\eta=\frac{1}{2} \sqrt{\frac{\rho \sigma}{k}}
$$

With the right viscosity of liquid 2, these capillary waves can be damped out in less than a millisecond, given that the wavelength is a millimeter or less. Thus, surface wave in a form of ripples will not pose any serious impediment on the dynamic operation of the LDM. Finding the suitable material for liquid 2 would not be a problem since there exist a lot of liquids having the required viscosity.

\section{PRELIMINARY MEASUREMENT}

To test the principle of the proposed device, we experimented on a $\mathrm{X}$ ray filter device ${ }^{8}$ which has the same actuation mechanism as discussed in Section 2.1. Fig. 3 shows the device immersed in a pool of two immiscible 


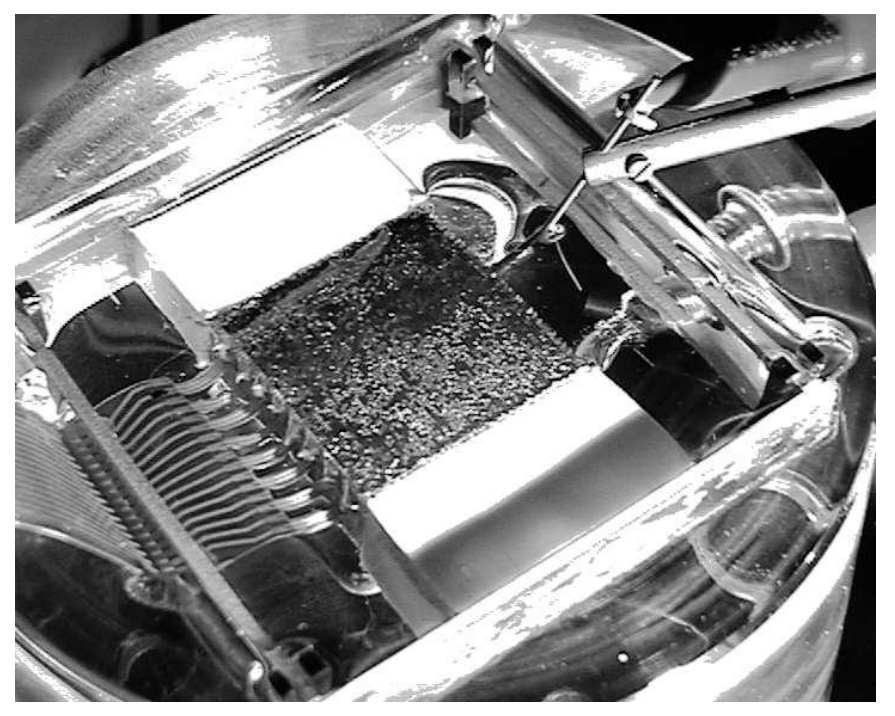

Figure 3. A $64 \times 64$ array of microchannels previously used as a $\mathrm{X}$ ray filter

liquids ( $\mathrm{NaCl}$ solution and $n$-Hexadecane). It consists of a $64 \mathrm{x} 64$ array of capillaries arranged in a hexagonal structure (see Fig. 5). Each individual capillary has a diameter of approximately $350 \mu \mathrm{m}$. The device is connected to a voltage source via a ribbon cable attached to the side wall of the structure. Electrical addressing of the array is done by grouping 8 capillaries (nearest neighbor) together in a column. Thus, there are 8 electrodes attached to the device. With this kind of addressing, switching an electrode results in the actuation of a column of capillaries instead of a single capillary. This is the obvious limitation of the device - it is not possible to measure the effect of actuating a single capillary. This limitation is addressed in the prototype LDM that is still under development. The X ray filter device can withstand high voltages (around $400 \mathrm{~V}$ ) before the voltage breakdown of the insulating material occurs. The typical operating voltage for liquid actuation is around $200 \mathrm{~V}$.

During the measurement, a thin layer of Hexadecane (approximately $2 \mathrm{~mm}$ ) sits on top of the microchannel. The floating reflective membrane was not used during the measurement. A pulse with an amplitude of $196 \mathrm{~V}$ was used. Other input signal waveforms (e.g., sine and sawtooth) were also applied onto the device.

The response time of the liquid deformable mirror was measured using the measurement set-up shown in Fig. 4. A beam from a laser diode is collimated using lens (L1). A portion of this beam is blocked by a knife edge $(\mathrm{K})$ and is focused onto the surface of the liquid by a second lens (L2). This beam is reflected back onto the second mirror (M2) and again recollimated using a third lens (L3). Any movement of the liquid surface results in a deflection of the beam. This deflection is detected by a fast photodetector. The beam deflection with respect to the vertical displacement of the liquid surface, in a planar state, was first calibrated to determine the actual height of the bump when the device is actuated.

\section{RESULTS AND DISCUSSION}

Fig. 6 shows the result obtained from the temporal measurement. A pulse with an amplitude of $196 \mathrm{~V}$ and duration of $100 \mathrm{~ms}$ (see Fig. 6a) was used as an input signal. The rise time of the liquid surface is depicted in Fig. 6b. The downward direction of the signal indicates the rise of the liquid while the upward direction denotes the depression. It can be observed from Fig. $6 \mathrm{~b}$ that the photodetector signal reaches its maximum before the end of the applied pulse. This maximum is then followed by a rippling movement, which indicates that surface waves were generated. The most important observation that can be made from the graph is that the maximum signal is reached at around $7 \mathrm{~ms}$ (see Fig. 6c). Using a calibration curve for the vertical displacement and the photodetector signal generated, this maximum photodetector signal corresponds to a displacement of about 1 $\mathrm{mm}$. One intriguing result, though, that needs further verification is that the liquid surface is depressed first 


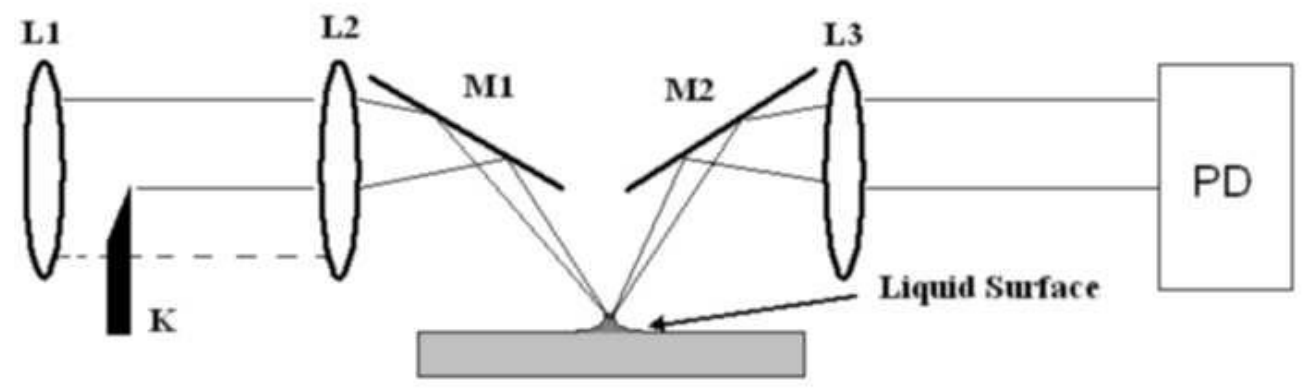

Figure 4. Measurement set-up used to determine the rise time of the liquid surface deformation.

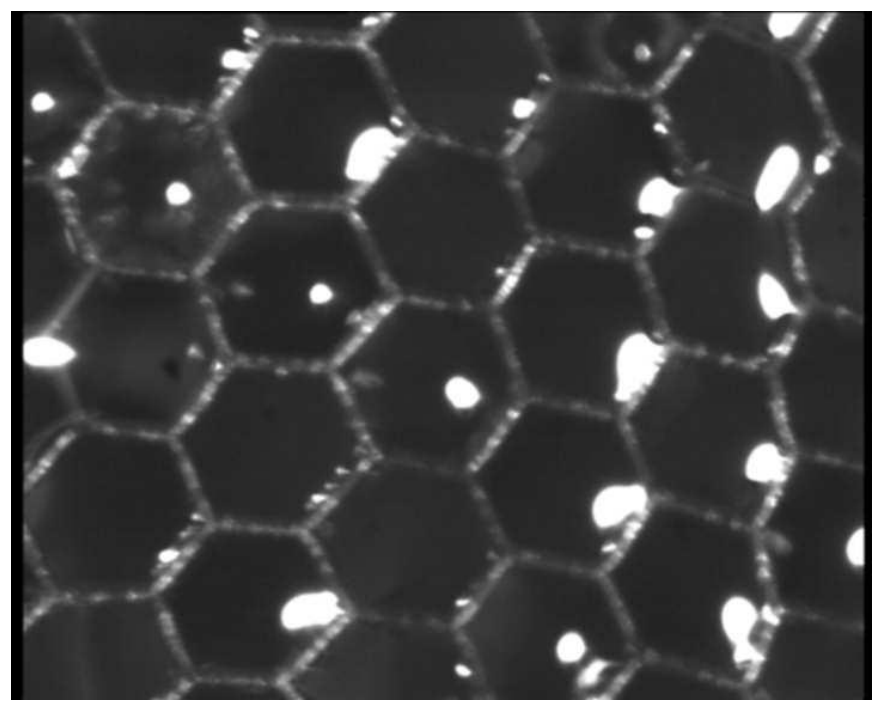

Figure 5. Top view of the honeycomb structure of microchannels filled with the dielectric liquid. The capillary diameter is about $350 \mu \mathrm{m}$. 


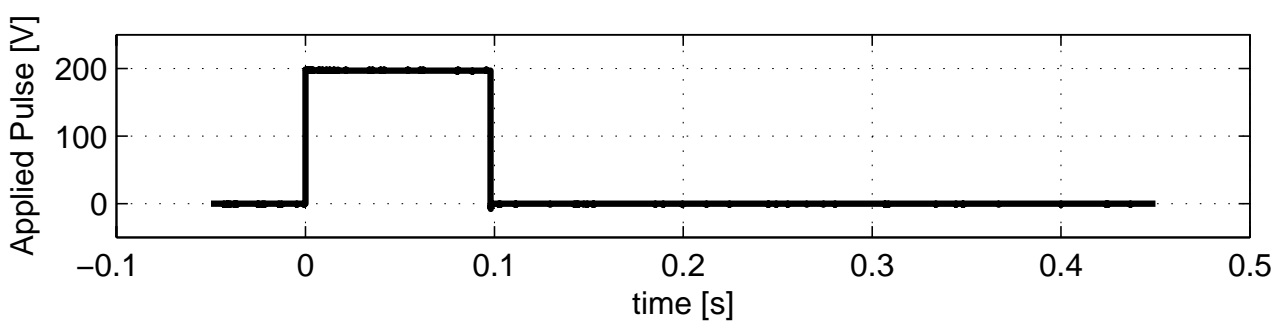

a)

time [s]

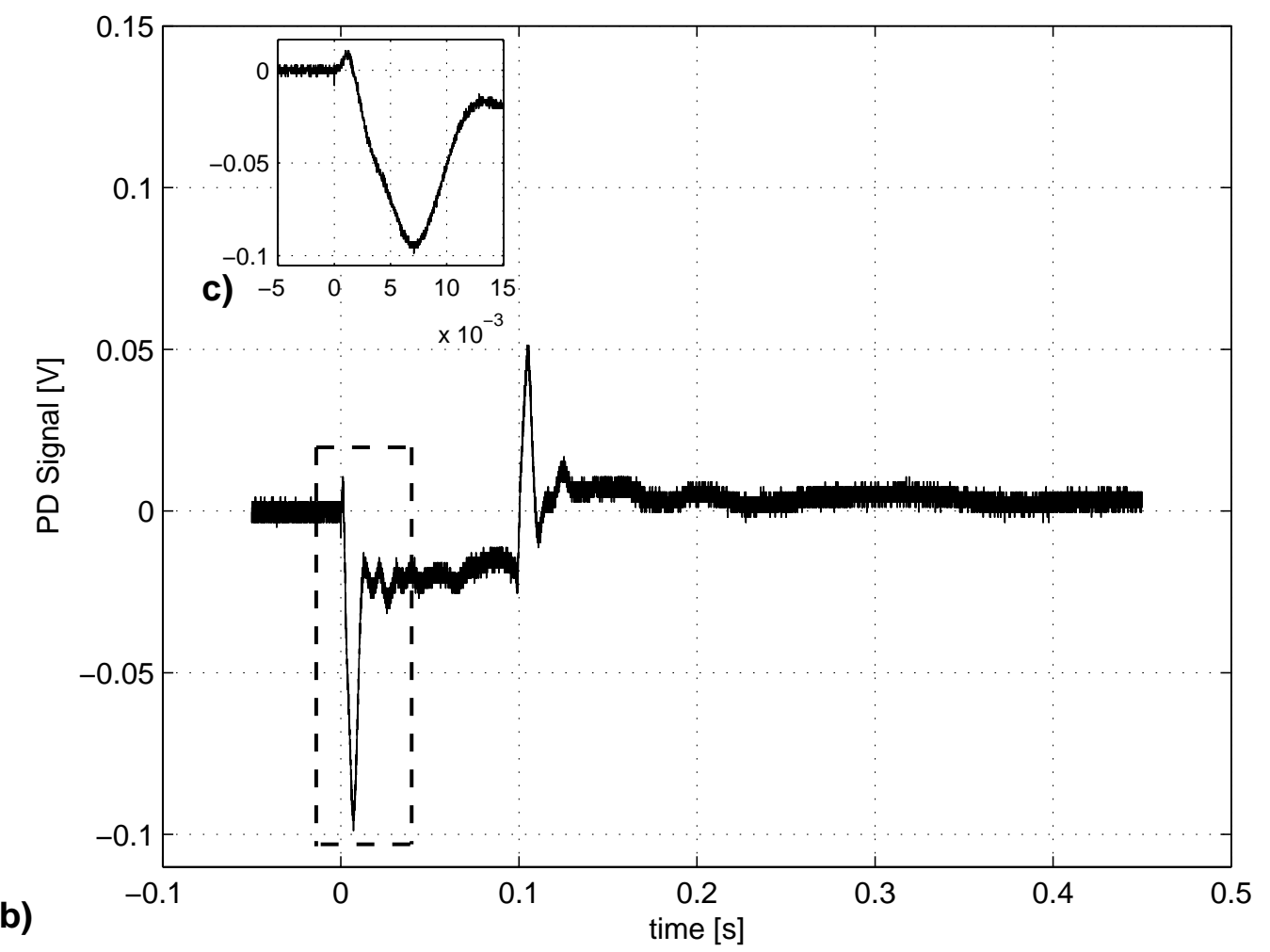

Figure 6. Rise time of the liquid surface due to an applied pulse with amplitude of $196 \mathrm{~V}$ and duration of $100 \mathrm{~ms}$. Inset (c) is the enlarged plot of the dotted region.

before creating a bump. This can be seen in Fig. 6c. The height of this depression is roughly $140 \mu m$ and is reached at approximately $1 \mathrm{~ms}$. The slope of the rise and fall of the surface bump is the same, as evident in the graph. This confirms that the time-scale of the upward and downward actuation are of the same magnitude. Surface ripples are present because the viscosity of the topmost liquid is not sufficient enough to fully damp the capillary waves. Hexadecane has a viscosity of $3.032 \mathrm{mPa} s$. A larger viscosity value for the top liquid is needed to fully extinguish the capillary waves. The observed rise time agrees well with the simulated electrocapillary rise time. The result obtained is based on the topmost liquid alone, without the thin floating membrane.

\section{CONCLUSION}

A novel deformable mirror based on liquid surface actuation by electrocapillary means is presented. With the use of a novel electrocapillary effect, the movement of liquid inside the capillary can be controlled. The proof of principle was validated using a $\mathrm{X}$ ray filter device. An initial measurement, using the $\mathrm{X}$ ray filter, of the rise time of the liquid surface deformation shows that the device has a fast response time in the order of 
several milliseconds. The proposed device offers several advantages such as high number of actuators, low power dissipation, and reduced fabrication cost.

\section{ACKNOWLEDGMENTS}

The authors are grateful for the generosity of M.W.J. Prins, at Philips Research, for the loan of the X ray filter device. This project is funded by the Dutch Organization for Applied Research (TNO) in the framework of the TNO-TU Delft Knowledge Center for Aperture Synthesis (KAS) .

\section{REFERENCES}

1. H. W. Babcock, "The possibility of compensating astronomical seeing," Astronomical Society of the Pacific 65, pp. 229-236, 1953.

2. F. V. Fischer and H. Thiemann, "Theoretische betrachtungen uber ein neues verfahren der fernsehgrossprojetion," Schweiz. Arch. Angew. Wiss. Tech. 7 (1), pp. 1-11, 1941.

3. R. Ragazzoni and E. Marchetti, "A liquid deformable mirror," Astron. Astrophys. 283, pp. L17-L19, 1994.

4. P. R. Laird, R. Bergamasco, V. Berube, E. Borra, J. Gingras, A. Ritcey, M. Rioux, N. Robitaille, S. Thibault, L. V. da Silva Jr., and H. Yockell-Lelievre, "Ferrofluid based deformable mirrors - a new approach to adaptive optics using liquid mirrors," in Adaptive Optical System Technologies II, P. Wizinowich and D. Bonaccini, eds., Proc. SPIE 4839, pp. 733-740, 2003.

5. F. Zamkotsian, H. Camon, N. Fabre, V. Conedera, and G. Moreaux, "Micro-deformable mirror for next generation adaptive optical systems," in Adaptive Optical System Technologies II, P. Wizinowich and D. Bonaccini, eds., Proc. SPIE 4839, pp. 711-720, 2003.

6. A. W. Adamson, Physical Chemistry of Surfaces, 5th edition, John Wiley \& Sons, New York, 1990.

7. W. J. J. Welters and L. G. J. Fokkink, "Fast electrically switchable capillary effects," Langmuir 14, pp. 15351538, 1998.

8. M. W. J. Prins, W. J. J. Welters, and J. W. Weekamp, "Fluid control in multichannel structures by electrocapillary pressure," Science 291, pp. 277-280, 2001.

9. K. Wang and T. Jones, "Electrowetting dynamics of microfluidic actuation," Langmuir 21, pp. 4211-4217, 2005.

10. L. Landau and E. M. Lifshitz, Fluid Mechanics, Butterworth-Heinemann, Burlington, Mass., 2003. 